\title{
A NOTE ON THE UNION-CLOSED SETS CONJECTURE
}

\author{
GIOVANNI LO FARO
}

(Received 10 July 1991; revised 20 January 1992)

Communicated by L. Caccetta

\begin{abstract}
It has been conjectured that for any union-closed set $\mathscr{A}$ there exists some element which is contained in at least half the sets in $\mathscr{A}$. It is shown that this conjecture is true if the number of sets in $\mathscr{A}$ is less than 25. Several conditions on a counterexample are also obtained,

1991 Mathematics subject classification (Amer. Math. Soc.): 05A05, 05A99.

Keywords and phrases: sets, union-closed.
\end{abstract}

\section{Introduction}

A union-closed set $\mathscr{A}$ is defined as a non-empty finite collection of distinct, non-empty finite sets, closed under union (that is, if $S \in \mathscr{A}$ and $T \in \mathscr{A}$ then $S \cup T \in \mathscr{A}$ ).

The following conjecture is rephrased from [1].

CONJECTURE. Let $\mathscr{A}=\left\{A_{1}, A_{2}, \ldots, A_{n}\right\}$ be a union-closed set. Then there exists an element which belongs to at least $\left\lceil\frac{n}{2}\right\rceil$ of the sets in $\mathscr{A}$, where

$$
\left\lceil\frac{n}{2}\right\rceil= \begin{cases}\frac{n}{2} & \text { if } n \text { is even } \\ \frac{n+1}{2} & \text { if } n \text { is odd. }\end{cases}
$$

In this paper we generalize results due to Sarvate and Renaud and to Norton and Sarvate. In particular we establish some inequalities involving the $A_{j}$ 's and $n$ which must hold for any counterexample and prove that the conjecture is valid when $n \leq 24$.

(c) 1994 Australian Mathematical Society 0263-6115/94 \$A2.00+0.00

This research was supported by G.N.S.A.G.A. (C.N.R.) and by M.U.R.S.T., Fondi $40 \%$. 


\section{Preliminaries and notations}

We denote the union-closed set $\mathscr{A}=\left\{A_{1}, A_{2}, \ldots, A_{n}\right\}$ by $\mathscr{A}(n)$. Assume, for convenience, that $\left|A_{i}\right|=w_{i}, w_{1} \leq w_{2} \leq \cdots \leq w_{n}=q$, and $A_{n}=I_{q}=\{1,2, \ldots, q\}$.

The support size of an $\mathscr{A}(n)$ is defined to be the number $q=w_{n}$. Let $\mathbb{A}(n, q)$ denote the set of possible $\mathscr{A}(n)$ with support size $q$. Theorems 2 and 3 in [4] show that the conjecture holds when $\mathscr{A}(n) \in \mathbb{A}(n, q), q \leq 6$.

Let $\mathscr{A}(n) \in \mathbb{A}(n, q)$ and let $x \in I_{q}$. Define $\mathscr{A}_{x}(n)$ to be the set of $A_{i}$ in $\mathscr{A}(n)$ which contain $x$ and let $\left|\mathscr{A}_{x}(n)\right|=d(x)$. Assume $\mathscr{C}_{x}(n)$ to be the set of $A_{j}$ in $\mathscr{A}(n)$ not containing $x$ and let $C_{x}=\cup\left\{A_{j}: A_{j} \in \mathscr{C}_{x}(n)\right\}$. Set $\mathscr{A}_{x}^{*}=\left\{A_{i}-\{x\}: A_{i} \in \mathscr{A}(n)\right\}$; it is clear that $\mathscr{A}_{x}^{*}$ is a union-closed set with support size $q-1$.

\section{Restrictions on the set sizes}

Theorem 2 in [5] shows that the conjecture holds whenever $w_{1}+w_{2} \geq q$. This can be improved by the following result:

THEOREM 1. The conjecture holds whenever

$$
\begin{array}{ll}
w_{3}+w_{\frac{n+3}{2}} \geq q, & \text { if } n \text { is odd } \\
w_{4}+w_{\frac{n+4}{2}} \geq q, & \text { if } n \text { is even } .
\end{array}
$$

PROOF. (i) Suppose $d(x) \leq(n-1) / 2$ for all $x \in I_{q}$. This implies that

$$
w_{1}+w_{2}+\cdots+w_{n-1}+q \leq \frac{n-1}{2} q
$$

and so

$$
\begin{aligned}
w_{1}+w_{2}+\left(w_{3}+w_{\frac{n+3}{2}}\right) & +\left(w_{4}+w_{\frac{n+5}{2}}\right)+\cdots+\left(w_{\frac{n+1}{2}}+w_{n-1}\right) \leq \\
& \leq w_{1}+w_{2}+\frac{n-3}{2}\left(w_{3}+w_{\frac{n+3}{2}}\right) \leq \frac{n-3}{2} q,
\end{aligned}
$$

hence $w_{3}+w_{\frac{n+3}{2}}<q$, a contradiction.

(ii) Theorem 1 in [4] shows the validity of the conjecture for odd $n$ leads to its validity for $n+1$, then the proof is similar to the previous case.

\section{Smallest counterexample}

Let $n_{0}$ be the minimum value of $n$ taken over all the counterexamples to the unionclosed conjecture. By Theorem 1 of [4], assume $n_{0}=2 t+1$. Let $\mathscr{A}\left(n_{0}\right) \in \mathbb{A}\left(n_{0}, q_{0}\right)$ 
be a counterexample to the conjecture with minimal support size $q_{0}$. We have the following:

THEOREM 2.

$$
\begin{aligned}
& \left|\mathscr{A}_{x}^{*}\right|<\left|\mathscr{A}\left(n_{0}\right)\right| \quad \text { for each } x \in I_{q_{0}} . \\
& \mathscr{A}_{x}\left(n_{0}\right) \neq \mathscr{A}_{y}\left(n_{0}\right) \quad \text { for each } x, y \in I_{q_{0}}, x \neq y \text {. }
\end{aligned}
$$

PROOF. (i) Suppose $\mathscr{A}_{x_{0}}^{*} \in \mathscr{A}_{x_{0}}\left(n_{0}, q_{0}-1\right)$ for some $x_{0} \in I_{q_{0}}$. By the minimality of $q_{0}$ there exists an element $z$ in $(t+1)$ sets of $\mathscr{A}_{x_{0}}^{*}$, hence in $(t+1)$ sets of $\mathscr{A}\left(n_{0}\right)$, a contradiction.

(ii) It is enough to consider that $\mathscr{A}_{x}\left(n_{0}\right)=\mathscr{A}_{y}\left(n_{0}\right)$ implies $\left|\mathscr{A}_{x}^{*}\right|=\left|\mathscr{A}_{y}^{*}\right|=$ $\left|\mathscr{A}\left(n_{0}\right)\right|$.

THEOREM 3. For any $x \in I_{q_{0}}$ there exists $A_{i} \in \mathscr{A}\left(n_{0}\right)$ such that $A_{i} \in \mathscr{A}_{x}\left(n_{0}\right)$ and $A_{i}-\{x\} \in \mathscr{C}_{x}\left(n_{0}\right)$.

PROOF. Suppose, on the contrary, there is $x_{0} \in I_{q_{0}}$ such that $A_{i}-\left\{x_{0}\right\} \notin \mathscr{A}\left(n_{0}\right)$ for every $A_{i}$ containing $x_{0}$ in $\mathscr{A}\left(n_{0}\right)$. This implies $\left|\mathscr{A}_{x_{0}}^{*}\right|=\left|\mathscr{A}\left(n_{0}\right)\right|$ contradicting (i) of Theorem 2.

COROLlaRY 1. For any $x \in I_{q_{0}}, C_{x} \cup\{x\} \in \mathscr{A}\left(n_{0}\right)$.

PROOF. Let $A_{i} \in \mathscr{A}_{x}\left(n_{0}\right)$ such that $A_{i}-\{x\} \in \mathscr{C}_{x}\left(n_{0}\right)$. Then $A_{i}-\{x\} \subseteq C_{x}$ and $C_{x} \cup A_{i}=C_{x} \cup\{x\} \in \mathscr{A}\left(n_{0}\right)$.

THEOREM 4. If $x, y \in I_{q_{0}}$, then $d(x) \leq d(y)$ implies $y \in C_{x}$.

PROOF. Suppose, on the contrary, $y \notin C_{x}$. For every $A_{i} \in \mathscr{A}_{y}\left(n_{0}\right)$ it follows that $x \in A_{i}$ (otherwise $y \in A_{i} \subseteq C_{x}$ ), hence $d(x)=d(y)$ and then $\mathscr{A}_{x}\left(n_{0}\right)=\mathscr{A}_{y}\left(n_{0}\right)$, contradicting (ii) of Theorem 2 .

CoROLlaRY 2. If $d(x)=\operatorname{Min}\left\{d(y): y \in I_{q_{0}}\right\}$ then $C_{x}=I_{q_{0}}-\{x\}$.

PROOF. Immediate from the theorem.

Let $x, y \in I_{q_{0}}$. By Theorem 4, $x \in C_{y}$ or $y \in C_{x}$ and so we have the following.

COROLlaRY 3. $C_{x} \neq C_{y}$, for every $x, y \in I_{q_{0}}$.

PRoOF. Assume $d(x) \leq d(y)$, then $y \in C_{x}$ and $y \notin C_{y}$. 
THEOREM 5. $n_{0} \geq 2 q_{0}+1$.

PROOF. In [2], Theorem 2.1, it is proved that, in any counterexample $\mathscr{A}=$ $\left\{A_{1}, A_{2}, \ldots, A_{n}\right\}$ to the conjecture with $n$ minimal, there are at least three distinct elements, each of which appears in exactly $(n-1) / 2$ of the $A_{j}$ 's. Assume $d\left(q_{0}\right)=\left(n_{0}-1\right) / 2$. By Theorem $3 q_{0} \in C_{i}$, for every $i=1,2, \ldots,\left(q_{0}-1\right)$, and $q_{0} \in I_{q_{0}}=A_{n_{0}}$. It follows that $q_{0} \leq\left(n_{0}-1\right) / 2$. This completes the proof.

THEOREM 6. There are at least three distinct elements $x_{1}, x_{2}, x_{3} \in I_{q_{0}}$ such that $C_{x_{i}}=I_{q_{0}}-\left\{x_{i}\right\}, i=1,2,3$.

PROOF. Let $d\left(x_{1}\right)=\min \left\{d(x): x \in I_{q_{0}}\right\}$. Corollary 2 implies that $C_{x_{1}}=I_{q_{0}}-\left\{x_{1}\right\}$. It is easy to see that there exists an $A_{i} \in \mathscr{A}_{x_{1}}\left(n_{0}\right)$ such that $A_{i} \neq I_{q_{0}}$. Let $A_{i}=$ $I_{q_{0}}-\left\{y_{1}, y_{2}, \ldots, y_{r}\right\}$, then $x_{1} \in A_{i} \subseteq C_{y_{1}} \subseteq I_{q_{0}}-\left\{y_{1}\right\}$. If $C_{y_{1}}=I_{q_{0}}-\left\{y_{1}\right\}$ we put $x_{2}=y_{1}$ otherwise $C_{y_{1}}=I_{q_{0}}-\left\{y_{1}, z_{1}, z_{2}, \ldots, z_{s}\right\}$ and so $x_{1} \in A_{i} \subseteq C_{y_{1}} \cup\left\{y_{1}\right\} \subseteq$ $C_{z_{1}}$. Obviously, continuing this process, we find an $x_{2} \in I_{q_{0}}\left(x_{2} \neq x_{1}\right)$ such that $C_{x_{2}}=I_{q_{0}}-\left\{x_{2}\right\}$. Let $A_{x_{i}}^{\prime}$ be a set of minimal cardinality containing $x_{i}(i=1,2)$. Certainly $A_{x_{1}}^{\prime} \cup A_{x_{2}}^{\prime} \neq I_{q_{0}}$, (otherwise, since $\mathscr{B}=\left\{A_{1}, A_{2}, \ldots, A_{n_{0}}\right\}-\left\{A_{x_{1}}^{\prime}, A_{x_{2}}^{\prime}\right\}$ is union-closed, there would exist an element $z$ in at least $t$ sets of $\mathscr{B}\left(n_{0}-2\right)$ and hence in at least $(t+1)$ sets of $\left.\mathscr{A}\left(n_{0}\right)\right)$. Arguing as above we can easily complete the proof.

Let $d(1)=\min \left\{d(x): x \in I_{q_{0}}\right\}$ and let $\mathscr{B}=\mathscr{A}\left(n_{0}\right)-\mathscr{A}_{1}\left(n_{0}\right)$. Obviously $\mathscr{B}$ is a union-closed set. For each $z \in I_{q_{0}}$, put $|\{B \in \mathscr{B}: z \in B\}|=d^{*}(z)$. Let $x_{1}=1, x_{2}, x_{3}$ be as in Theorem 6 . We have the following.

THEOREM 7. $d(1) \geq 5$.

Proof. Obviously $d(1) \geq 3$. Suppose $d(1)=4$. By Theorem 6, we have $\mathscr{A}_{1}\left(n_{0}\right)=\left\{I_{q_{0}}, I_{q_{0}}-\left\{x_{2}\right\}, I_{q_{0}}-\left\{x_{3}\right\}, B_{1}\right\}$ and so there exists a $z \in I_{q_{0}}-\{1\}$ with $d^{*}(z) \geq\left(n_{0}-3\right) / 2$, hence $d(z)=\left(n_{0}+1\right) / 2$, a contradiction.

THEOREM 8. $C_{x_{4}} \supseteq I_{q_{0}}-\left\{x_{3}, x_{4}\right\}$,for some $x_{4} \in I_{q_{0}}, x_{4} \notin\left\{x_{1}, x_{2}, x_{3}\right\}$.

PROOF. Case (1) Either $A_{x_{1}} \cup A_{x_{2}} \subset I_{q_{0}}-\left\{x_{3}\right\}$ or $A_{x_{1}} \cup A_{x_{3}} \subset I_{q_{0}}-\left\{x_{2}\right\}$ for some $A_{x_{i}} \in \mathscr{A}_{x_{i}}\left(n_{0}\right), i=1,2,3$. Without loss of generality, we may suppose that $A_{x_{1}} \cup A_{x_{2}} \subset I_{q_{0}}-\left\{x_{3}\right\}$. Proceeding as in Theorem 6, we get that $A_{x_{1}} \cup A_{x_{2}} \subseteq C_{x_{4}}$ and $C_{x_{4}} \supseteq I_{q_{0}}-\left\{x_{3}, x_{4}\right\}$.

Case (2) Note that $A_{x_{1}} \cup A_{x_{2}} \supseteq I_{q_{0}}-\left\{x_{3}\right\}$ and $A_{x_{1}} \cup A_{x_{3}} \supseteq I_{q_{0}}-\left\{x_{2}\right\}$ for all $A_{x_{i}} \in \mathscr{A}_{x_{i}}\left(n_{0}\right), i=1,2,3$. We prove that Case (2) is not possible. Let $A_{x_{i}}^{\prime}$ be a set of minimal cardinality containing $x_{i}, i=1,2,3$. For $j=2,3$, let $z_{j}$ be an element of $I_{q_{0}}$ which belongs to at least $\left(n_{0}-1\right) / 2$ of the sets in $\mathscr{A}\left(n_{0}\right)-\left\{A_{x_{1}}^{\prime}, A_{x_{j}}^{\prime}\right\}$. It is easy 
to see that $z_{2}=x_{3}$ and $z_{3}=x_{2}$ and so $d\left(x_{2}\right)=d\left(x_{3}\right)=\left(n_{0}-1\right) / 2$. By Theorem 7 there exists $x_{4} \notin\left\{x_{1}, x_{2}, x_{3}\right\}$ such that $x_{4} \notin A_{x_{1}}^{\prime}$. Since $A_{x_{1}}^{\prime} \cup A_{x_{2}} \supseteq I_{q_{0}}-\left\{x_{3}\right\}$ for each $A_{x_{2}} \in \mathscr{A}_{x_{2}}\left(n_{0}\right)$, it follows that $\mathscr{A}_{x_{2}}\left(n_{0}\right) \subseteq \mathscr{A}_{x_{4}}\left(n_{0}\right)$, hence $\mathscr{A}_{x_{2}}\left(n_{0}\right)=\mathscr{A}_{x_{4}}\left(n_{0}\right)$ contradicting Theorem 2 .

Let $I_{q_{0}}=\left\{1,2, \ldots, q_{0}\right\}$ with $d(1) \leq d(2) \leq \cdots \leq d\left(q_{0}\right)$. By Theorem 4 and Corollary 3 , it follows that $\left|C_{s}\right| \in\left\{w_{1}, w_{2}, \ldots, w_{n_{0}}\right\},\left|C_{s}\right| \geq q_{0}-s$ and $w_{n_{0}-s} \geq q_{0}-s$, for each $s \in I_{q_{0}}$. Theorems 6 and 8 say that $w_{n_{0}-4} \geq q_{0}-2, w_{n_{0}-3}=w_{n_{0}-2}=w_{n_{0}-1}=$ $q_{0}-1$ and so, by a similar argument to that used in Theorem 1 , we can prove the following.

COROLlaRY 4. $w_{10}+w_{\frac{n_{0}+3}{2}}<q_{0}$.

PROOF. Note that

$$
\begin{aligned}
w_{1}+w_{2}+\cdots+w_{9}+\cdots+w_{n_{0}-8} & +\left(q_{0}-7\right)+\left(q_{0}-6\right)+\left(q_{0}-5\right)+ \\
& +\left(q_{0}-2\right)+3\left(q_{0}-1\right)+q_{0} \leq \frac{n_{0}-1}{2} q_{0} .
\end{aligned}
$$

Then

$$
\left(w_{1}+\cdots+w_{9}\right)+\frac{n_{0}-17}{2}\left(w_{10}+w_{\frac{n_{0}+3}{2}}\right)-23 \leq \frac{n_{0}-17}{2} q_{0},
$$

hence $w_{10}+w_{\left(n_{0}+3\right) / 2}<q_{0}$.

THEOREM 9. $d(1) \geq 9$.

PROOF. By Theorem 7, we need to consider the following cases.

Case $d(1)=5$. Let $\mathscr{A}_{1}\left(n_{0}\right)=\left\{I_{q_{0}}, I_{q_{0}}-\left\{x_{2}\right\}, I_{q_{0}}-\left\{x_{3}\right\}, B_{1}, B_{2}\right\}$, with $I_{q_{0}}-$ $\left\{x_{3}, x_{4}\right\} \subseteq B_{1} \subseteq I_{q_{0}}-\left\{x_{4}\right\}$, and let $z \in I_{q_{0}}-\{1\}$ such that $d^{*}(z)=\left(n_{0}-5\right) / 2$. Necessarily, $B_{1}=I_{q_{0}}-\left\{x_{3}, x_{4}\right\}, z=x_{3}, x_{3} \notin B_{2}$ and $d\left(x_{3}\right)=\left(n_{0}-1\right) / 2$. Since $x_{4} \in\left(I_{q_{0}}-\left\{x_{3}, x_{4}\right\}\right) \cup A_{x_{3}}$, for each $A_{x_{3}} \in \mathscr{A}_{x_{3}}\left(n_{0}\right)$, it follows that $\mathscr{A}_{x_{3}}\left(n_{0}\right) \subseteq \mathscr{A}_{x_{4}}\left(n_{0}\right)$, hence $\mathscr{A}_{x_{3}}\left(n_{0}\right)=\mathscr{A}_{x_{4}}\left(n_{0}\right)$, contradicting Theorem 2 .

Case $d(1)=6$. Similar to the previous case.

Case $d(1)=7$. Let $\mathscr{A}_{1}\left(n_{0}\right)=\left\{I_{q_{0}}, I_{q_{0}}-\left\{x_{2}\right\}, I_{q_{0}}-\left\{x_{3}\right\}, B_{1}, B_{2}, B_{3}, B_{4}\right\}$ with $I_{q_{0}}-\left\{x_{3}, x_{4}\right\} \subseteq B_{1} \subseteq I_{q_{0}}-\left\{x_{4}\right\}$ and let $z \in I_{q_{0}}-\{1\}$ such that $d^{*}(z)=\left(n_{0}-7\right) / 2$. Necessarily $z \in\left\{x_{2}, x_{3}, x_{4}\right\}$.

Suppose $B_{1}=I_{q_{0}}-\left\{x_{4}\right\}$, then $z \notin B_{2} \cup B_{3} \cup B_{4}$. We can assume $z=x_{2}$. If there exists $B_{i}(i=2,3,4)$ such that $y \notin B_{i} \cup\left\{x_{2}, x_{3}, x_{4}\right\}$, then $B_{i} \cup A_{x_{2}} \supseteq I_{q_{0}}-\left\{x_{3}\right\}$ or $B_{i} \cup A_{x_{2}} \supseteq I_{q_{0}}-\left\{x_{4}\right\}$ and so $y \in A_{x_{2}}$, hence $\mathscr{A}_{y}\left(n_{0}\right)=\mathscr{A}_{x_{2}}\left(n_{0}\right)$, a contradiction.

If $B_{i} \supseteq I_{q_{0}}-\left\{x_{2}, x_{3}, x_{4}\right\}$, for each $i=2,3,4$, then

$$
\begin{aligned}
\mathscr{A}_{1}\left(n_{0}\right)=\left\{I_{q_{0}}, I_{q_{0}}-\left\{x_{2}\right\}, I_{q_{0}}-\left\{x_{3}\right\}, I_{q_{0}}-\left\{x_{4}\right\},\right. \\
\left.I_{q_{0}}-\left\{x_{2}, x_{3}\right\}, I_{q_{0}}-\left\{x_{2}, x_{4}\right\}, I_{q_{0}}-\left\{x_{2}, x_{3}, x_{4}\right\}\right\} .
\end{aligned}
$$


Let $A_{x_{2}}^{\prime}$ be a set of minimal cardinality containing $x_{2}$. Obviously $\mathscr{B}=\mathscr{A}\left(n_{0}\right)-$ $\left\{\mathscr{A}_{1}\left(n_{0}\right) \cup\left\{A_{x_{2}}^{\prime}\right\}\right.$ is a union-closed set and so there exists an $r$ in $\left(n_{0}-7\right) / 2$ sets of $\mathscr{B}$, a contradiction.

Suppose $B_{1}=I_{q_{0}}-\left\{x_{3}, x_{4}\right\}$ and, obviously, $I_{q_{0}}-\left\{x_{4}\right\} \notin \mathscr{A}_{1}\left(n_{0}\right)$.

Let $z=x_{2}$. Necessarily $x_{2} \notin B_{2} \cup B_{3} \cup B_{4}, d\left(x_{2}\right)=\left(n_{0}-1\right) / 2$ and there exists a $B_{i}(i=2,3,4)$ such that $y \notin B_{i} \cup\left\{x_{2}, x_{3}, x_{4}\right\}$, otherwise

$\mathscr{A}_{1}\left(n_{0}\right)=\left\{I_{q_{0}}, I_{q_{0}}-\left\{x_{2}\right\}, I_{q_{0}}-\left\{x_{3}\right\}, I_{q_{0}}-\left\{x_{3}, x_{4}\right\}, I_{q_{0}}-\left\{x_{2}, x_{3}\right\}, I_{q_{0}}-\left\{x_{2}, x_{4}\right\}, I_{q_{0}}-\left\{x_{2}, x_{3}, x_{4}\right\}\right\}$.

and so $\left(I_{q_{0}}-\left\{x_{2}, x_{4}\right\}\right) \cup\left(I_{q_{0}}-\left\{x_{3}, x_{4}\right\}\right)=I_{q_{0}}-\left\{x_{4}\right\} \notin \mathscr{A}_{1}\left(n_{0}\right)$. We again obtain $\mathscr{A}_{y}\left(n_{0}\right)=\mathscr{A}_{x_{2}}\left(n_{0}\right)$, which is a contradiction.

Let $z=x_{4}$. This is similar to the case $z=x_{2}$.

Let $z=x_{3}$. Necessarily $d\left(x_{3}\right) \geq\left(n_{0}-3\right) / 2$. Since $I_{q_{0}}-\left\{x_{3}, x_{4}\right\} \cup A_{3}=I_{q_{0}}$, it follows that $\mathscr{A}_{x_{3}} \subset \mathscr{A}_{x_{4}}$. If $d\left(x_{3}\right)=\left(n_{0}-1\right) / 2$ then $\mathscr{A}_{x_{3}}\left(n_{0}\right)=\mathscr{A}_{x_{4}}\left(n_{0}\right)$, a contradiction. If $d\left(x_{3}\right)=\left(n_{0}-3\right) / 2$ then $d\left(x_{4}\right)=\left(n_{0}-1\right) / 2$ and $I_{q_{0}}-\left\{x_{3}\right\}$ is the only set of $\mathscr{A}\left(n_{0}\right)$ containing $x_{4}$ but not $x_{3}$ and so $\left|\mathscr{A}_{x_{3}}^{*}\left(n_{0}\right)\right|=n_{0}-1$. Let $r \in I_{q_{0}}$ such that $r$ is contained in $\left(n_{0}-1\right) / 2$ sets of $\mathscr{A}_{x_{3}}^{*}\left(n_{0}\right)$. Obviously $r \in I_{q_{0}}-\left\{x_{3}\right\}$, hence $d(r)=\left(n_{0}+1\right) / 2$, a contradiction.

Case $d(1)=8$. Similar to the previous case.

Let $d_{r}=\left|\left\{x \in I_{q_{0}}: d(x)=r\right\}\right|$. Obviously $\sum d_{r}=q_{0}$. For each element $x_{i}$ counted in $d_{9}$ we have $C_{x_{i}}$ containing $I_{q}-\left\{x_{i}\right\}$ by Theorem 4 , hence $\left|C_{x_{i}}\right|=q_{0}-1$. For each $x_{i}$ contained in $d_{10}$ we have $C_{x_{i}}$ containing all elements $x_{j}$ such that $d\left(x_{j}\right) \geq 10$ except $x_{i}$ itself, hence $\left|C_{x_{i}}\right| \geq q_{0}-d_{9}-1$. Counting in this way, Theorem 2 of [4] and Theorem 9 lead to this inequality:

$$
\begin{aligned}
q_{0}+d_{9}\left(q_{0}-1\right)+d_{10}\left(q_{0}-1-d_{9}\right)+d_{11}\left(q_{0}-1-d_{9}-d_{10}\right)+\cdots+ \\
+d_{\frac{n_{0}-1}{2}}\left(q_{0}-1-d_{9}-d_{10}-\cdots-d_{\frac{n_{0}-3}{2}}\right)+3\left(n_{0}-q_{0}-1\right) \leq \\
\leq 9 d_{9}+10 d_{10}+\cdots+\frac{n_{0}-1}{2} d_{\frac{n_{0}-1}{2}}
\end{aligned}
$$

and then

$$
q_{0}^{2}-3 q_{0}+3\left(n_{0}-1\right)-\sum d_{i} d_{j} \leq 9 d_{9}+10 d_{10}+\cdots+\frac{n_{0}-1}{2} d_{\frac{n_{0}-1}{2}} .
$$

THEOREM 10. $n_{0} \geq 25$.

PROOF. Theorem 4 in [5] shows that $n_{0} \geq 19$.

For $n_{0}=19,\left(^{*}\right)$ leads to $q_{0}^{2}-12 q_{0}+54 \leq 0$ which never holds.

For $n_{0}=21,\left(^{*}\right)$ gives $q_{0}^{2}-\left(13+d_{9}\right) q_{0}+\left(d_{9}^{2}+d_{9}+60\right) \leq 0$ which never holds. 
For $n_{0}=23,\left(^{*}\right)$ gives:

$$
q_{0}^{2}-\left(14+d_{9}+d_{10}\right) q_{0}+\left(d_{9}^{2}+d_{10}^{2}+d_{9} d_{10}+2 d_{9}+d_{10}+66\right) \leq 0
$$

which never holds.

This completes the proof of the theorem.

\section{References}

[1] 'A much travelled conjecture', Austral. Math. Soc. Gaz., 14 (1987), 63.

[2] R. M. Norton and D. G. Sarvate, 'Union-closed sets conjecture; some observations', unpublished manuscript, College of Charleston, 1989.

[3] J-C. Renaud, 'Is the union-closed sets conjecture the best possible?', J. Austral. Math. Soc. (Ser. A) 51 (1991), 276-283.

[4] D. G. Sarvate and J-C. Renaud, 'On the union-closed sets conjecture', Ars Combin. 27 (1989), 149-153.

[5] - 'Improved bounds for the union-closed sets conjecture', Ars Combin. 29 (1990), 181-185.

[6] P. Winkler, 'Union-closed sets conjecture', Austral. Math. Soc. Gaz. 14 (1987), 99.

Dipartimento di Matematica

Università di Messina

Contrada Papardo-Salita Sperone, 31

98166 Sant'Agata

Messina

Italy 